\title{
Féeries
}

Études sur le conte merveilleux, XVII ${ }^{\mathrm{e}} \mathrm{XIX}{ }^{\mathrm{e}}$ siècle

$4 \mid 2007$

Le conte, la scène

\section{Le féerique moral dans les contes moraux de Marmontel}

Nicolas Veysman

\section{(2) OpenEdition}

1 Journals

Édition électronique

URL : http://journals.openedition.org/feeries/413

DOI : $10.4000 /$ feeries.413

ISSN : 1957-7753

Éditeur

UGA Éditions/Université Grenoble Alpes

Édition imprimée

Date de publication : 1 octobre 2007

Pagination : 213-234

ISBN : 978-2-84310-101-4

ISSN : 1766-2842

Référence électronique

Nicolas Veysman, "Le féerique moral dans les contes moraux de Marmontel », Féeries [En ligne], 4| 2007, mis en ligne le 04 novembre 2008, consulté le 08 septembre 2020. URL : http://

journals.openedition.org/feeries/413; DOI : https://doi.org/10.4000/feeries.413

Ce document a été généré automatiquement le 8 septembre 2020

(c) Féeries 


\title{
Le féerique moral dans les contes moraux de Marmontel
}

\author{
Nicolas Veysman
}

“ J'AI GRAND REGRET DE LA FÉERIE, disait Marmontel en 1757. C'étoient pour les imaginations vives une source de plaisirs innocens et la manière la plus honnête de faire d'agréables songes ». L'homme mûr, alors âgé de 34 ans, exprime-t-il ici sa nostalgie pour un âge révolu pendant lequel les « bisaïeuls » racontaient au petit Jean-François des « contes merveilleux ${ }^{1} »$ ? C'est possible, mais on observera qu'en l'occurrence le merveilleux est moins féerique que moral, que les premiers souvenirs du mémorialiste sont moins la douce réminiscence d'une féerie enfantine que la mémoire d'un enchantement vertueux. Le regret marmontélien de la féerie serait donc moins une confidence autobiographique qu'une déploration littéraire : ce sont les mots d'un conteur ; mais le conteur déplore-t-il vraiment la disparition des fées? L'inventeur du conte moral ne s'en félicite-t-il pas plutôt, secrètement ? Tout porte à croire que Marmontel est moins attristé par la disparition du conte de fées qu'occupé à promouvoir le " conte moral » dont on lui attribue la paternité ; son regret n'est que l'expression antiphrastique d'une appropriation de la féerie par le conte moral, sous la forme nouvelle d'un « féerique moral ».

2 Un mot sur les contes moraux. Jean-François Marmontel en a écrit un grand nombre, vingt-trois entre 1755 et 1765 - dont les premiers furent publiés dans le Mercure de France qu'il dirigea entre 1755 et 1758 -, dix-sept autres après 1790, appelés « nouveaux contes moraux ", parus dans le même périodique et, comme les premiers, réunis en recueil ; soit quarante contes moraux parmi lesquels ne figure qu'un seul véritable conte de fées, Les Quatre Flacons, publié en juillet 1757. Il s'agit du cinquième récit destiné à enrichir le Mercure dans lequel Marmontel a déjà inséré Le Moi (1755), Soliman II (mars 1756), Le Scrupule (juillet 1756) et Tout ou rien (décembre 1756). Nous disons « récit » et non "conte moral ", car, à cette date, Marmontel n'écrit pas de "contes moraux " : Le Moi est une " histoire très ancienne ", Soliman II une " anecdote turque ", Le Scrupule un simple " conte " et Tout ou rien une " anecdote moderne", tandis que le conte de fées de juillet 1757 n'est affublé d'aucune indication générique - les mots du 
titre suffisent à déterminer son inscription. À l'instar de toutes les autres fictions brèves, il ne deviendra "conte moral " qu'en 1761, lorsque l'auteur les réunit en recueil. Avant cette date, seules Les Deux Infortunées avait reçu pareille appellation précédemment employée par Crébillon dans Le Sopha (1740) puis par l'abbé de Voisenon dans Il eut raison (1755).

3 Il est important de préciser que lors de ce baptême tardif Marmontel ne procède à aucune réécriture et parfois même maintient les sous-titres originaux. De fait, le conte marmontélien est une production hybride, à la fois conte moral et conte de fées, mais aussi conte moral et conte libertin (Alcibiade), conte oriental (Soliman II), conte pastoral (Palémon) et bien d'autres choses encore. Tous ces mariages, même les plus improbables, sont possibles et nous nous proposons ici d'étudier celui qui associe la morale et la féerie.

4 L'union est ancienne, puisque Perrault faisait de la moralité un trait générique des « histoires du temps passé » - qu'il érigeait comme le modèle populaire du conte de fées - grâce auquel le conte moderne se distingue de la fable antique. La morale du conte de fées n'est toutefois pas celle du conte moral. En un demi-siècle, de 1690 à 1757 , c'est moins le message qui change que les conditions et les modalités de la moralité humaine. On ne comprendra cette évolution qu'en rappelant comment le conte moral est né de la progressive dislocation entre féerie et morale dans le conte de fées à la fin du XVII ${ }^{e}$ siècle et lors des premières décennies du siècle suivant. De sa naissance jusqu'à son déclin, de l'équivoque native jusqu'aux dérives libertines, le conte de fées est en effet traversé par une tension entre l'extravagance féerique et l'édification morale ${ }^{2}$ qui dessine deux voies majeures qu'emprunteront les conteurs. Parmi ceux qui choisissent de restaurer la visée morale du conte, Fénelon, Caylus, Leprince de Beaumont et bien d'autres encore, qui ouvrent la voie au conte moral.

Trois contes féeriques

5 Le conte moral se souvient du conte de fées, de la féerie euphorique des premières années après son invention à la fin du XVII ${ }^{e}$ siècle mais aussi de la féerie critique qui lui succède, jusqu'à la critique de la féerie et sa dissolution dans le politique ${ }^{3}$. Marmontel n'écrit qu'un conte de fées, Les Quatre Flacons, mais il faut aussi prendre en considération les éléments féeriques présents dans d'autres contes moraux, dans Le Mari Sylphe (1765) et dans Les Promenades de Platon, nouveau conte moral dont la publication fut posthume.

6 Les Quatre Flacons d'abord : Alcidonis, jeune homme de Mégare, né sous la protection de la fée Galante " qui le doua du don de plaire, sans aucun penchant décidé à l'amour ", est envoyé par son père à Athènes pour y connaître le monde. Il le met en garde contre les préjugés sur les femmes et affirme qu'il existe partout des femmes honnêtes capables de rendre heureux un homme vertueux. Il fera plusieurs rencontres successives avec Séliane, Corine et Céphise, puis Thélésie, au cours desquelles il se met sous l'influence magique des flacons donnés par la Fée, le pourpre inspirant la passion absolue, le rose la fantaisie inconstante, le bleu le goût vertueux, tandis que le blanc lui permet de revenir à son naturel. Alcidonis ne trouvera le bonheur que dans l'union morale avec Thélésie.

$7 \quad$ Les Quatre Flacons sont un véritable conte de fées, avec son personnel convenu, sa scène rituelle et inaugurale des dons au cours de laquelle un nouveau-né se voit attribuer ses principales qualités. Marmontel apporte cependant d'importantes modifications au

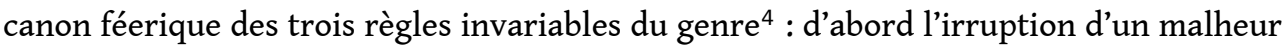


- sous la forme d'une malédiction lancée par une mauvaise fée, qui trouve toujours une entière réparation à la fin du conte : à la force aveugle du destin est préférée la proclamation de la liberté humaine et des bienfaits de l'expérience dont la fée est une précieuse auxiliaire - ressemblante au « Démon familier de Socrate » que Marmontel range parmi les génies d'antan, «intelligences médiatrices entre les hommes et les Dieux »; ensuite l'excellence du personnage principal auquel sont distribuées les plus hautes vertus : Marmontel est bien peu loquace sur ses qualités : on apprend que « sa jeunesse ne fut que le développement des talens et des grâces qu'il avoit reçus en partage » mais on ne saura rien de plus ; enfin l'intervention du merveilleux qui ouvre et clôt la fiction : le flacon bleu apporte en effet la " félicité » à notre jeune héros mais c'est de tous les artifices le moins merveilleux des quatre, en ce sens qu'il produit une rupture moins violente entre son naturel et ses sentiments magiques; non pas " changement prodigieux » du flacon pourpre ni « révolution » inconcevable du flacon blanc, ni même la vive agitation produite par le flacon couleur de rose - " voilà ses yeux et ses désirs qui se promènent sans se fixer ». Le pouvoir magique et son effet sur Alcidonis va s'affaiblissant et c'est sans surprise qu'on découvre « la chaleur douce et vive » que répand dans les « veines » d'Alcidonis le flacon bleu.

Le Mari Sylphe ensuite : au sortir du couvent où elle vivait d'illusions romanesques, Élise épouse le marquis de Volange auquel elle ne témoigne que de la froideur, tandis qu'elle se montre très sensible à la fiction des Sylphes. Pour guérir sa femme de telles chimères, le mari, avec la complicité d'une servante, endosse nuitamment le rôle de pur esprit et multiplie les prodiges, jusqu'à ce que le sylphe Valoé qui a résolu de s'incarner en Volange mette en vain à l'épreuve la fidélité de la rêveuse mais vertueuse Élise.

9 Le Mari Sylphe n'est pas un conte de fées : inséré dans le recueil de 1765, c'est bel et bien un conte moral. Il est certes émaillé de motifs féeriques, comme en témoigne le lexique : Élise est « enchantée » ou bien victime d'« enchantement " à la vue d'une « merveille " ou d'un "prodige inconcevable "; il est même question de " fées " et de " génie " pour expliquer les mystères qui se reproduisent autour d'elle. Toutefois ces mots sanctionnent toujours un point de vue erroné sur le monde ou bien constituent une allusion ironique à la mystification dont Élise est l'objet. Ainsi Volange à sa femme qui découvre au petit matin une robe semblable à celle qui, la veille, avait attiré son attention : «En vérité, poursuivit-il, en considérant de plus près l'étoffe, cela est fait de main de Fées. Cette façon de parler familière venoit là si à propos, qu'élise rougit comme si on l'eût trahie, et que son secret eût été révélé. » Il y a donc bien recul de la féerie, non par amenuisement mais par inversion de la merveille : la féerie n'est plus un phénomène surnaturel mais un état d'âme forgé par un habile prestidigitateur à côté duquel le lecteur est invité à s'amuser des naïfs émerveillements de l'héroïne. Anticonte de fées, par conséquent, où le féerique, comme chez Fénelon ou Caylus ${ }^{5}$, est retourné contre lui-même pour constituer la matière d'un spectacle comique et de propos ironiques.

10 Théâtralité et ironie sont les symptômes d'une subversion du conte de fées par l'introduction d'une variation de la focalisation : en alternant régulièrement les points de vue, Marmontel réduit l'enchantement féerique à l'illusion d'une vision personnelle abusée par un malicieux stratagème ; la féerie cesse d'exercer son empire sur les esprits dès qu'elle est relativisée. C'est proprement un préjugé, selon la définition qu'en donne Michel Delon :

La critique cartésienne du préjugé peut déboucher sur deux attitudes: la condamnation du préjugé comme non-raison, voire déraison, ou bien relativisation 
des raisons de chacun, l'établissement d'une typologie des points de vue ou la reconnaissance d'une genèse du raisonnement comme jamais achevé. Le préjugé est rejeté comme incapacité d'accéder au vrai ou bien expliqué comme aspect particulier et relatif d'une vérité complexe ${ }^{6}$.

11 Déraison ou raison relative : deux façons de concevoir le préjugé, deux façons aussi de penser la merveille féerique, deux façons enfin de procéder au désenchantement. Élise est moins folle que recluse dans une perspective exclusive qui fomente ses illusions et la vérité lui apparaîtra lorsqu'en toute fin du conte elle croisera son regard sur la féerie avec celui de son mari et celui de sa confidente. La jeune femme suit l'évolution du siècle qui désigne le préjugé non plus comme "pure erreur " mais comme " particularisme, métaphore ou limitation » et le pense dorénavant comme le " moment d'un devenir ${ }^{7}$ ». Au terme de son parcours, Élise sera, il est vrai, parvenue à changer la féerie merveilleuse en féerie morale : "C'est à présent, dit Élise, en se jetant dans les bras de son mari, c'est à présent que je suis enchantée, et j'espère que la mort seule détruira cet enchantement ».

La féerie n'est plus dans Le Mari Sylphe ce qu'elle était dans Les Quatre Flacons ; remède utile aux maladresses d'une jeunesse inexperte, elle constitue à présent un mal que seule l'ingéniosité du mari philosophe saura guérir. Elle était un salutaire auxiliaire dans la recherche du parfait amour; elle devient un obstacle au bonheur vertueux, comme c'est aussi le cas dans le dernier des nouveaux contes moraux, long récit découpé en sept parties où la féerie tient une place très discrète.

Les Promenades de Platon : au terme de ses pérégrinations en Sicile pendant lesquelles il restaure les amitiés compromises et venge l'innocence outragée, Platon fait à Denis le Jeune le récit de ses bienfaits. Au monarque qui se plaint de sa difficulté à connaître la vérité, le philosophe répond par un conte merveilleux : le roi Darius demande au sage Aspase de découvrir les causes de la mélancolie du prince Archèménès. On apprend qu'au cours de ses voyages le prince rencontra l'un des derniers mages qui lui accorda un privilège : utiliser un anneau lui permettant de lire dans toutes les âmes. Le prince, averti des pensées de tous ceux qui l'entourent, sombre alors dans la mélancolie et ne sera guéri qu'en buvant l'eau d'oubli.

Les Promenades de Platon constituent la fin d'un parcours que les trois contes moraux auront suffi à suggérer : après la douce euphorie de l'initiation féerique à l'amour, après la distance critique induite par la démystification philosophique, Marmontel prône une sage renonciation aux pouvoirs surnaturels de l'omniscience; après le ministère traditionnel de la fée Galante, après la comédie du Sylphe, le conteur met en scène l'un des derniers représentants d'un peuple jadis massacré. Par delà la progressive extinction $\mathrm{du}$ personnage féerique, sa fonction change notablement : il incarne d'abord en 1757 la lucidité sentimentale, puis en 1765 une ingénieuse sagacité philosophique et enfin dans le dernier des contes moraux une funeste clairvoyance politique. La féerie est devenue une maladresse, comme le dit le philosophe au mage :

[...] je ne trouve pas moins injuste qu'imprudent ce que vous avez fait pour lui ; car l'avantage de lire dans les cœurs est pour un homme, quel qu'il soit, un privilége intolérable. Achéménès n'en a point abusé ; mais le droit d'en user, même innocemment, n'appartient à aucun être sous le ciel ; en le lui confiant, vous avez fait de lui le plus redoutable des hommes, et moi-même je l'aurais fui, s'il l'avait gardé plus long-temps ; car autant il m'est doux d'avoir un Dieu pour témoin et pour juge de mes plus secrètes pensées, autant il me serait pénible et dur que, sans mon propre aveu, un mortel en fût confident. 
Et le mage de convenir de son inconséquence :

Vous avez bien raison, dit le mage en souriant avec douceur ; mais à mon âge on est si faible! au sien l'on est si séduisant! Allons, le mal est fait, il faut tâcher de l'adoucir. Retournons dans mon cabinet remettre l'anneau à sa place ; car dans ce moment il m'afflige en me faisant lire à moi-même dans le cœur du fidèle Aspase le juste et sensible reproche d'avoir rendu son ami malheureux.

Comme dans Les Quatre Flacons et dans Le Mari Sylphe, la féerie est congédiée et, une fois encore, le conte s'achève sur un prodige moral : «O Dieu ! s'écria-t-il, quelle révolution soudaine, quel prodige s'opère en moi ! Ah ! je sors d'un pénible songe ! Et qu'avez vous rêvé ? lui demanda le mage. Je n'en sais rien, répondit le prince ; mais je sais que j'ai bien souffert. » De fait, la féerie est devenue illicite, mais sait-on ce qui a rendu les fées hors-la-loi ? Il est peu probable que la seule pensée philosophique ait conduit Marmontel, avec " grand regret ", à donner congé aux fées et aux génies d'antan. Nous avons vu que dès 1757 la féerie entre en lice avec la morale et que la tutelle du bon père le dispute à celle de la fée prodigue. Dès son entrée dans le conte marmontélien, la féerie est contrainte de s'astreindre aux règles de la morale, pour devenir, de merveilleuse qu'elle était, une féerie morale.

De la féerie morale à la morale féerique

17 Féerie morale ou morale féerique ? En vérité, les deux ensemble, ou plutôt l'une puis l'autre, d'abord la féerie morale, présente dans les trois contes que nous avons évoqués, puis la morale féerique, commune à tous les contes, anciens et nouveaux, contemporains et posthumes, car l'une et l'autre constituent les deux étapes d'un même processus d'assimilation de la féerie par le conte moral.

18 Féerie morale puis morale féerique, par conséquent. Le chiasme qui croise les catégories grammaticales indique quelles modifications littéraires sont intervenues au cours de cette évolution : la féerie perd la fonction rectrice de noyau du groupe nominal pour devenir un adjectif dont l'accord et le sens dépendent du nouveau substantif. Le maître est devenu l'esclave au terme d'une sourde belligérance entre féerie et morale.

Dans Les Quatre Flacons se côtoient en effet la féerie traditionnelle et une analyse morale inspirée de la "nouvelle préciosité " du premier xviII siècle où sont envisagées les différentes formes du sentiment amoureux. S'il y a préséance de la féerie sur la morale, elle est bien illusoire ; le recours de Marmontel à l'allégorie prouve même le contraire, puisque l'avantage accordé au merveilleux s'explique par la transparence d'un sens littéral derrière lequel l'auteur donne à lire une signification seconde. En effet, la mise en forme allégorique du conte établit une hiérarchie inverse à l'ordre des apparences dans laquelle la vérité morale, à chercher par delà la lettre féerique du texte, constitue le propos véritable de l'auteur. Certains, comme Crébillon, se plaisent à le dissimuler ; d'autres en revanche, comme Marmontel, se font une règle de le rendre évident. Dans Les Quatre Flacons, la visibilité de ce fonds moral est proportionnelle à l'inconsistance de la féerie : la « fée Galante » est insipide à souhait, en accord avec la fonction vicariante qu'elle remplit dans le conte. Comment pourrait-il en être autrement, quand le dispositif allégorique lui-même n'a de légitimité poétique qu'en s'astreignant à une exacte transparence morale : « Le mérite de l'apologue est de cacher le sens moral ou la vérité qu'il renferme, jusqu'au moment de la conclusion, qu'on appelle moralité ", écrit Marmontel dans ses Éléments de littérature, tandis que « le mérite de l'allégorie est de 
n'avoir pas besoin d'expliquer la vérité qu'elle enveloppe ; elle la fait sentir à chaque trait par la justesse de ses rapports ${ }^{8} »$.

La féerie est donc l'organe mineur du dispositif allégorique ; c'est un voile en charge d'orner de ses charmes la morale sérieuse du foyer domestique. C'est encore le cas dans Le Mari Sylphe où la féerie égaie le propos moral mais, en 1765, elle le fait aux dépens d'elle-même : on ne rit plus avec les fées mais contre elles; on ne s'abandonne plus à la fable joyeuse de la magie amoureuse, on détruit avec jubilation le mensonge du merveilleux.

De toute évidence, dans la féerie morale, le registre entre en conflit avec le genre, et l'antagonisme ne disparaît qu'au terme d'une réduction de la féerie au féerique, c'est-àdire au terme de la réduction du genre du conte de fées à la dimension d'un registre littéraire, conjointement à l'accession de la morale au rang de genre littéraire avec l'invention du conte moral. On ne parlera plus alors de féerie morale mais de morale féerique.

La morale féerique n'est plus seulement l'apanage de quelques contes; elle est un principe invariant valable pour tous les contes. À ce titre, tous les contes moraux de Marmontel sont peu ou prou des contes féeriques, c'est-à-dire des contes de fées sans fées, desquelles le conteur aura conservé plusieurs éléments fondamentaux nécessaires à la constitution du genre nouveau : le déroulement narratif et la présence du prodige.

Le déroulement narratif d'abord : le conte moral s'approprie, en l'aménageant à ses exigences propres, le cadre fictionnel du conte de fées. C'est vrai en particulier de la structure temporelle : même imprécision historique, même référence au temps des origines. Certes le lecteur reconnaît l'époque, ancienne ou contemporaine, mais aucune indication datée ne vient inscrire chronologiquement l'histoire. Peu importe finalement l'époque, parce que le conte moral évoque un moment et une expérience intemporelle : le départ de l'âge d'or. Le conteur évoque une sortie de l'ingénuité par une prise de conscience du monde dans lequel le personnage est sommé d'entrer : féerique ou moral, le conte est toujours l'histoire d'une rupture parenthétique qui confronte le héros à la difficulté d'une épreuve à surmonter en un délai limité. Récit d'une socialisation, le conte moral s'inscrit dans une temporalité de l'urgence et de la crise entourée, en amont de l'incipit et en aval de la clausule, par la temporalité contraire d'un bonheur durable, celui primitif de l'enfance et celui conquis de la vie maritale. Le personnage est ainsi partagé entre l'exigence de conquérir un bonheur nouveau et la nostalgie d'un retour vers l'âge d'or. Le merveilleux se déploie et le prodige moral s'accomplit promptement dans ce moment transitoire entre la paisible inconscience de l'enfance et la sereine assurance de l'union vertueuse. Comme le conte de fées, le conte moral est une variation qui opère, après l'épreuve d'un désordre moral, un retour à la vertu initiale et plus encore à un état antérieur à cette vertu native, puisque la morale n'est véritable que si elle est la conversion sociale effective d'une virtualité naturelle.

24 La présence du prodige ensuite : dans sa Poétique française, Marmontel établit une distincte importante entre " deux sortes de merveilleux, l'un en deçà, l'autre au-delà des limites de la nature " qu'il nomme «merveilleux naturel » et " merveilleux surnaturel »- même si, dans le même mouvement Marmontel s'applique à les confondre comme imitation de la nature ${ }^{9}$. Parmi les exemples du « merveilleux naturel pris [...] sur la dernière limite des possibles ${ }^{10}$ " que mentionne Marmontel, un seul est compatible avec la simplicité du conte mais il constituera une donnée invariante de 
tous les contes moraux : l'excellence des vertus et la grandeur de caractère. Elles motivent des comportements extrêmes et appartiennent à ce que nous appellerons le " merveilleux moral » dont chaque conte est une illustration : « Tels sont par conséquent les caractères et les génies d'une force, d'une vigueur, d'une élévation extraordinaires ".

Entendons-nous bien: l'heure n'est plus aux surenchères précieuses du portrait aristocratique ni aux naïves perfections du conte de fées. Ce prodige-là est congédié dès 1755 , dans les premières lignes du premier conte moral : « La nature et la fortune sembloit avoir conspiré au bonheur d'Alcibiade. Richesses, talens, beauté, naissance, la fleur de l'âge et de la santé ; que de titres pour n'avoir tous les ridicules! Alcibiade n'en avoit qu'un : il vouloit être aimé pour lui-même. » Le prodige véritable est moral et c'est toujours le fait d'une vertu exceptionnelle.

Marmontel mettra du temps avant de trouver une formule narrative qui mette en relief cette vertu sublime; il lui faudra abandonner la structure épisodique et sa liste précieuse des différents types de sentiments amoureux pour se tourner vers une logique tensive en 1758, dans son premier " conte moral ». " La structure n'est plus épisodique, mais se resserre autour d'un conflit d'opinions ou de caractères ${ }^{11}$ ", écrit Kate Astbury pour qui la rupture déterminante se produit en effet dans Les Deux Infortunées où est sensible l'influence d'une littérature anglaise dont Marmontel fait l'éloge dans son Mercure.

L'évolution est déterminante parce qu'elle préside à l'émergence du prodige moral qui réside, selon Marmontel, dans une tension entre deux modalités différentes voire contradictoires de l'énergie humaine : la force morale et la vivacité sentimentale. Lorsque l'une et l'autre se concilient, se produit un miracle moral que ne perce pas l'esprit humain : « Je ne conçois pas encore l'empire que ce jeune cœur avoit pris sur lui-même pour me cacher son mal ", dit-on dans La Veillée. La pensée religieuse impose encore ses cadres et son vocabulaire sacré pour approcher le prodige moral et passionnel, mais ne nous y trompons pas ; c'est bien par les seules vertus de son origine naturelle et de son existence sociale que l'homme accède à la sainteté vertueuse. Le sublime n'est plus une sortie de l'humain; il est devenu une expérience des limites de l'humanité : «Quelle fut ma vertu! ou plutôt quelle fut la force que me donna la crainte de la faire expirer si je me dévoilois! Je l'eus cette force incroyable ». Dans les contes, le symptôme de l'extrême puissance de cet élan est la dépression mélancolique dans laquelle sombre l'homme vertueux, et le péril de mort physique auquel il s'expose : le désespoir et la mort sont les premiers salaires d'une vertu et d'une sensibilité exceptionnelles qui atteignent les limites morales aussi bien que sentimentales. Les deux surenchères sont indissociables dans le conte moral. La morale religieuse et l'immoralisme libertin n'accèdent jamais à cette grandeur parce qu'ils rompent la symétrie en subordonnant soit les passions humaines à leur réprobation morale, soit les convenances sociales à la licence sexuelle. Toutes les deux se méprennent sur la nature humaine : la morale religieuse ignore la dimension passionnelle de la moralité humaine et l'immoralisme libertin la sublimation morale des pulsions. L'une et l'autre désunissent et opposent ce que la nature humaine concilie. Seule la morale philosophique et son illustration littéraire préservent l'équilibre du couple en procédant à une double surenchère, gageant qu'une exaltation des passions est le meilleur et le seul garant d'une incomparable intégrité morale. Équivalent laïc du miracle et de la sainteté vécus ailleurs comme une sortie surnaturelle et contemplative 
du monde, le prodige moral et naturel est aussi un événement social : le héros moral suit un parcours d'exception au cœur de l'espace social et ses relations avec autrui jouent un rôle prépondérant. Nous distinguerons deux types de liaisons sociales propices au prodige moral : la résistance vertueuse à la débauche et la conversion morale du vicieux. Marmontel illustre la première dans L'École de l'amitié où la vertu d'une femme fait face à la dépravation de son mari : « Je regarde comme un prodige la jeune femme qui résiste aux séductions d'un époux vicieux, s'il en est aimé »; la seconde dans Les Solitaires de Murcie où les opinions d'une femme vertueuse parviennent à réformer $\mathrm{M}$. de Villarcé qui constate lui-même sa métamorphose morale : « J'ai rougi à mes propres yeux ; et dès lors j'ai été changé. Vous l'êtes prodigieusement, reprit ma sœur ».

Les fées ont disparu et il incombe dorénavant à la vertu de faire des miracles, et si le conte moral apparait comme "un petit drame qui finit bien, la résolution verbale, toujours gaie et spirituelle, d'un faux conflit ${ }^{12}$ ", c'est au conte de fées qu'il le doit, à sa propension à finir heureusement et à celle de surmonter les obstacles par le seul pouvoir des mots. La merveille aura lieu dans le conte moral, mais conformément aux seules lois de la nature ; elle sera naturelle mais, comme c'était aussi le cas dans le conte de fées, ne sera pas soumise à l'exigence classique de la vraisemblance : la merveille sera à la fois naturelle et invraisemblable. Marmontel lui donne un nom, le « prodige » :

Si la nature, s'éloignant de ses sentiers battus, produit un composé moral ou physique assez étrange, pour nous persuader qu'elle y a mis une expresse intention de se surpasser elle-même, ou de ne pas se ressembler ; ce procédé, dont les moyens nous sont inconnus, nous étonne, et devient un prodige à nos yeux. Voilà donc dans la nature même une sorte de merveilleux, connu sous le nom de prodiges $^{13}$.

L'idée n'est pas nouvelle et le XVII e siècle, en la personne de Chapelain, s'est déjà efforcé d'imposer contre l'invraisemblance merveilleuse la catégorie intermédiaire d'un vraisemblable extraordinaire ${ }^{14}$. Dans le cadre spécifique des fictions brèves, la légitimité de l'extravagance est d'actualité lors de la querelle de La Princesse de Clèves qui, après celle ressemblante $\mathrm{du}$ Cid, révèle la crise de la vraisemblance comme catégorie normative de la fiction littéraire à la fin du XVII siècle. Une solution a été trouvée par l'invocation d'une vraisemblance interne, c'est-à-dire purement textuelle, qui fonde la crédibilité d'un événement fictif sur sa nécessité diégétique : sera vraisemblable ce que le texte a rendu narrativement incontournable, et non ce qui s'accorde avec l'opinion commune. « La preuve est faite, par l'exemple fictif, écrit Marc Escola, que l'événement sans exemple n'est pas inimaginable, que la fiction peut être ce monde possible où le lecteur fera l'expérience de ce qu'il croyait impossible ${ }^{15}$ ", de sorte que "la fiction est lieu où le possible advient comme nécessaire ». Le prodige marmontélien sera donc naturel, invraisemblable et nécessaire ${ }^{16}$. Mais n'oublions pas l'essentiel : il sera surtout moral. Non pas moral et irrationnel, comme c'était le cas dans les contes de fées; non pas rationnel et immoral, comme l'est l'aveu dans $L a$ Princesse de Clèves ; le prodige marmontélien sera rationnel et moral.

Une grave difficulté point cependant. Comment concilier l'invraisemblance du prodige avec l'exemplarité morale du conte ? comment penser ensemble l'énonciation morale d'une loi générale et la narration de ce qui est sans exemple et par conséquent échappe à toute généralisation ? Le problème est insoluble en termes classiques. On sait que la nouvelle a résolu la contradiction en s'affranchissant de la forme de l'exemplum. Le conte n'y parvient qu'au prix d'une révision de la notion de vraisemblance fictionnelle. 
Marmontel s'y applique à partir de 1763 dans sa Poétique française puis plus tard dans ses Éléments de littérature, comme l'a montré Jan Herman dans un bel article sur la résolution marmontélienne du « dilemme du roman » :

Le but immédiat de la fiction est de persuader. La persuasion est inséparable de la vraisemblance. Cette vraisemblance, qu'elle soit externe ou interne, se définit comme le respect d'un modèle intérieur et universel que la Nature a gravé dans nos âmes en nous donnant l'idée de ce qui est beau, de ce qui est bon et de ce qui est vrai. Or, de même qu'au niveau de la vraisemblance, une mutation esthétique fonde la représentation poétique dans un modèle intérieur, de même Marmontel tentera d'enraciner la moralité de l'œuvre dans un tel modèle général ${ }^{17}$.

32 La vraisemblance devient l'expression fictionnelle d'une réalité morale idéale, conforme au modèle intérieur. Que deviennent la vraisemblance externe et la vraisemblance interne? Elles ne sont elles-mêmes que l'expression d'une adéquation de la fiction avec ce patron moral : l'opinion commune, régie par la loi morale, ne tardera à valider le prodige moral, tandis que l'agencement textuel trouve en l'ordre moral une puissante source d'inspiration. "Dès que la narration est d'accord avec ellemême, et vraisemblable dans tous les points, il ne s'agit plus d'examiner ce qu'elle a de réel, pour savoir ce qu'elle a d'utile ", écrit Marmontel dans son Essai sur les romans.

La vraisemblance morale se confond avec le merveilleux naturel conçu comme actualisation fictionnelle des limites du possible et avec la moralité considérée comme émergence du modèle de vertu inscrit en chaque homme. Or la moralité n'est jamais aussi puissante que lorsque la fiction s'éloigne d'une vraisemblance externe, c'est-àdire de la conformité au réel, pour viser une vraisemblance interne; alors le conte, genre propice à cette distanciation à l'égard du réel, apparait comme favorable à une approche du modèle intérieur : libre de tourner le dos au réel, le conteur est, plus de tout autre, apte à approcher le modèle intérieur de la moralité. La fuite dans l'invraisemblance merveilleuse n'est pas une maladresse de moralisateur ni un travers maniériste ; c'est la condition d'une entrée dans la moralité de la fiction.

C'est pourtant de la maladresse que l'on impute à celui « qui ne fut ni tragique, ni poète, ni romancier et qui aurait tellement voulu l'être ${ }^{18}$ ", à celui qui occupa, dit-on, " un petit coin littéraire dans ce siècle de géants ${ }^{19}$ ". Les critiques sont parfois bien inconséquents et bien peu scrupuleux lorsqu'ils disent du mal de ceux qui ne seront pas oubliés après eux. On ne pardonne pas aux contes de Marmontel une " grâce mièvre, une simplicité calculée, un "naturel" tout de convention, qui fait qu'on n'y croit guère " mais on ne se demande pas ce qui conduit Marmontel à promouvoir l'invraisemblance morale.

\section{La fée Littérature}

« Chez lui, écrit Jean Sgard, le précepte est bien antérieur au récit ; il le soutient et le guide. Le conte devient l'évocation plaisante d'une situation fausse, composée en vue de sa résolution ». C'est si vrai que bien souvent le conteur place à l'initiale de la fiction un prologue qui formule dès l'abord la teneur morale du conte. Rien de provocateur dans ce geste qui, en dissipant toutes les attentes du lecteur par la divulgation du dénouement, constitue un élément déterminant du contrat de lecture.

Une fois encore, c'est la notion de vraisemblance qui permet de comprendre les choix poétiques de Marmontel dans ses contes moraux. Gérard Genette a montré que la vraisemblance classique réside dans la possibilité de rapporter des événements particuliers «à un corps de maximes reçues comme vraies par le public » et il stipule que « ces maximes, du fait même qu'elles sont admises, restent le plus souvent 
implicites ». Le récit vraisemblable repose sur cette reconnaissance muette qui fonde la crédibilité de la fiction et conforte l'illusion. En proférant la maxime, en exhibant sa source, Marmontel transgresse la loi du silence sur le fonctionnement du récit dont il propose au lecteur d'explorer les coulisses. Ce contrat de lecture comme « spectacle de l'invention » est propre à l'esthétique du conte ; c'est aussi celui du contes de fées à propos duquel Jean-Paul Sermain écrit qu'il "s'oppose au roman contemporain qui dissimule le rôle de l'écrivain soit derrière le paravent de l'histoire, soit derrière l'écriture d'un personnage qui prend pour matière sa propre existence, passée ou présente $^{20} »$. Quand il ne cède pas aux sirènes de l'illusion romanesque, comme cela lui arrive souvent dans ses contes, Marmontel s'adonne volontiers à maintenir une distance critique entre son invention et le lecteur ainsi initié aux arcanes de la création littéraire. C'est le cas dans les trois contes féeriques que nous avons étudiés, où Marmontel propose à son lecteur trois différentes incursions dans l'atelier du conteur.

Revenons d'abord aux Quatre Flacons. Nous savons qu'en 1757 le conte moral est encore en gestation et toute l'attention va alors au conte de fées dont Marmontel, sur le mode métafictionnel, va donner une définition oblique en la personne de la Fée Galante mais aussi et surtout à travers les autres personnages féminins du conte. À commencer par Séliane : on sait que cette femme "dans sa jeunesse, avoit été jolie et belle : elle étoit belle encore ; mais elle commençoit à n'être que jolie ». Derrière le dépérissement physique on reconnaît sans peine le déclin du conte de fées. L'idée est corroborée par les relations que la femme entretient avec son mari : contre l'homme qui incarne le principe de réalité, en rappelant à son épouse la vertu du combat et la valeur de l'argent, Séliane invoque les charmes du « beau monde ». Pour toute réponse, l'homme lui reproche son inutilité et sa folie, puis se retire. L'allusion est transparente : le réel, prosaïque et violent, est congédié par la féerie qui forme avec lui un bien «singulier ménage » : " Dès qu'il m’ennuie, je le contredis », déclare Séliane ; " il s'impatiente et se retire. L'on en fait tout ce qu'on veut. Je vous conseille de lui marquer de la déférence. Son amitié n'est pas à négliger : cela est bon à quelque chose. " Le couple incarne les relations tumultueuses entre la merveille et le vraisemblable qui occupe le débat poétique sur la fiction à l'Âge classique ${ }^{21}$. L'enjeu est de taille, puisqu'il s'agit de savoir quelle sera la poétique du conte moral. Sera-ce une poétique de l'extravagance ou bien une poétique de la véridicité ? On a vu que Marmontel parvient à concilier vérité et féerie à la faveur d'une mise en scène du prodige moral dans le monde moderne de l'Ancien Régime, mais le mari réaliste est néanmoins là pour mettre en garde le jeune homme contre les charmes dangereux des femmes et de la féerie - on sait que les contes de fées sont assimilés à une production littéraire essentiellement féminine - : «Gardez-vous bien, non pas d'elle, car son temps est passé, grâce au ciel ; mais gardez-vous de ses semblables. Ce sont les sirènes les plus dangereuses! Nulle sûreté dans leur commerce. Cela vous prend, vous trompe, et vous quitte sans pudeur. "C'est aux sirènes romanesques que le mari fait ici allusion : la mise en garde contre la féerie devient un préservatif contre le romanesque dans son ensemble, voire contre toutes les formes de fiction littéraire. La famille du jeune homme est elle-même frappée par le soupçon :

Vous tenez de votre pieux pere. Le bon homme ne juroit que par sa chaste moitié. C'étoit par complaisance pour lui qu'elle se paroit \& voyoit du monde ; par piété, qu'elle s'enfermoit avec un jeune Prêtre de Minerve; par recueillement, qu'elle alloit passer les soirées dans une petite maison qu'il avoit arrangée lui-même : il s'endormoit sur sa vertu de la meilleure foi du monde. - Il avoit raison, sans doute ; $\&$ je vous prie de respecter la mémoire de ma mere. - Ta mere ! ta mere étoit une 
femme : ne veux-tu pas qu'on l'eût faite exprès ? J'en ai bien vu ! je ne connois que mon extravagante qui soit exactement fidele ; \& encore est-ce moi qui l'ai formée. Je l'ai rendue vertueuse en dépit d'elle-même ; mais je n'ai pu lui ôter ce fonds de coquetterie, que la nature ou l'exemple leur inspire presqu'en naissant. Je gage qu'elle est capable encore de chercher à te séduire, pour le plaisir de se moquer de toi. $\mathrm{Tu}$ ne serois pas le premier qu'elle auroit mis au désespoir. Elle s'amusoit autrefois à ce petit jeu-là, \& puis elle m'en faisoit des contes, dont elle rioit comme une folle. Heureusement elle vieillit, \& le danger n'est plus si grand. féminine : Séliane incarne l'extravagance des fées anciennes dont les contes sont le témoignage de leurs folies romanesques et plus loin dans Les Quatre Flacons, elle renvoie à la littérature amoureuse. La fée Galante est toute autre ; fée morale, elle est l'organe d'une sagesse sociale qui prescrit tempérance et tolérance au jeune homme :

La Fée Galante lui apparut en songe, \& lui dit : Rien ne ressemble tant aux hommes que les femmes. Tout le bien, tout le mal qu'on en publie, est vrai en particulier, \& faux en général. Il ne faut, ni se fier à tout, ni se défier de tout. Vivez avec les femmes, mais ne vous y livrez qu'à propos. Je ne vous ai point donné de caractere, afin que vous soyez plus flexible au leur. Un homme décidé est un homme insociable. Vous serez charmant, si l'on dit de vous : on en fait tout ce qu'on veut. Mais ce n'est pas assez de plaire, il faut encore sçavoir aimer, \& n'aimer ni trop, ni trop peu.

cette fée morale, Alcidonis recevra quatre flacons qui seront autant de vertus et c'est grâce à elle que notre héros fera la conquête de la vertueuse Thélésie. Le conte de fées s'achève sur le triomphe de la vertu : « La vertu est la reine du monde ", y lit-on. Aux prodiges féeriques voués à la déception amoureuse succède le prodige moral :

Si vous n'avez un cœur d'airain, vous serez touché, Seigneur, du désespoir d'une infortunée qui donneroit son sang pour la liberté de son pere. Ariste, mon époux, à qui je n'avois pas rougi d'avouer que j'étois née d'un Esclave, n'a rien épargné pour rendre mon pere à mes vœux. Il l'a fait chercher vainement. J'apprends enfin qu'il est en votre pouvoir, \& je l'apprends dans l'indigence. J'ai apprécié tout ce qui me reste. Hélas ! il s'en faut bien que je sois en état de suffire à ce que vous exigez. Je n'ai plus qu'une seule ressource : c'est de m'offrir moi-même en échange pour mon pere. Il n'est pas juste que je sois libre, tandis que mon pere est esclave. Je suis jeune ; il est accablé d'années. Vous pouvez tirer de ma servitude plus d'avantages que de la sienne. Mes mains s'endurciront au travail ; mon cœur est fait à la patience. Si je voulois user de la facilité qu'on peut avoir à mon âge de séduire \& d'intéresser les hommes, je ne serois pas réduite à cette cruelle extrémité ; mais l'esclavage est moins honteux que le vice. Je n'hésite pas à choisir.

Dans ces quelques lignes, Marmontel dessine le patron de ce que seront tous les contes moraux à venir. Il y affirme la grandeur prodigieuse de la vertu éprouvée, la grandeur morale du sacrifice secret d'une fille pour son père. Le conte moral est à naître, confiné à l'intérieur d'une fiction féerique moribonde dont il s'alimente, comme une larve se nourrit d'un cadavre. La cloche sonne, mais personne ne sait si l'on célèbre un enterrement ou un baptême. Les Quatre Flacons sont la promesse d'une naissance ; c'est le premier cri de la vertu dans un conte né sous la protection d'une Fée lui promettant un radieux avenir :

Soyez heureux, Alcidonis, aimez sans inquiétude ; possédez sans dégoût ; desirez pour jouir ; faites des jaloux, \& ne le soyez jamais. Ce n'est pas un conseil que je vous donne ; c'est votre destin que je vous annonce. Vous avez bu à la source de la félicité parfaite. Je distribue à pleines mains des flacons pourpre \& couleur de rose ; mais le flacon bleu est un don que je réserve à mes favoris. 

douceurs de la vertu. La fée comble le vœu du père :

Mon cher fils, vous allez trouver dans le monde une foule de jeunes évaporés, qui se répandent en injures contre les femmes. N'en croyez rien. Ceux-là n'affectent de les mépriser, que parce qu'ils n'ont pu parvenir à les rendre méprisables. Pour moi, à commencer par votre mere, ma vertueuse épouse, j'ai reconnu dans le beau sexe une délicatesse de sentiment, une candeur, une vérité dont peu d'hommes sont capables. Faites comme moi, choisissez une femme honnête, d'une humeur égale, d'un caractere solide, d'une vertu sociable \& douce. Il y en a par-tout. Mon aveu suivra votre choix. Je suis bon pere : je ne veux que votre bonheur.

Passé le charme d'une si heureuse naissance, Marmontel portera un tout autre regard sur la féerie surnaturelle des contes anciens. L'heure est venue à la chenille de dévorer sa gangue : le conte moral revient en 1765 sur la féerie pour en faire le procès. On est d'abord étonné par les reprises lexicales d'un conte à l'autre. Ici et là, il est question de " songes » : songe grâce auquel le fée Galante s'adresse à son protégé ou bien songe pendant lequel Élise entre en relation avec son Sylphe. En 1757 comme en 1765 il sera question d'« imagination »:

\section{Les Quatres Falcons}

J'ai grand regret de la féerie. C'étoit pour les imaginations vives une sources de plaisirs innocens, et la manière la plus honnête de faire d'agréables songes.

Le Mari Sylphe

Lorsqu'Elise sortit du Couvent pour aller à l'Autel épouser le Marquis de Volange, elle étoit bien persuadée qu'après un amant, l'être le plus dangereux de la nature étoit un mari. Elevée par une de ces solitaires dont l'imagination mélancolique se peint en noir tous les objets [...].

On aura remarqué que les "imaginations vives », propices aux plaisirs innocents de la rêverie, ont laissé place à l'« imagination mélancolique ». La " folle du logis » a changé de visage :

Les Quatres Falcons

Seigneur, pardonnez : ma femme est folle. Je vous laisse ; car je n'y tiens plus. Par Hercule, Madame, faut-il que je sois votre mari !

Le Mari Sylphe

Je ne sçais quelle inquiétude lui prit, mais le soir ayant trouvé Justine un moment seule : Il me semble, lui dit-il, que ta maîtresse a eu bien du plaisir au spectacle ? Ah! Monsieur, elle en est folle.

La folie est devenue dangereuse, parce qu'elle compromet dorénavant le bonheur auquel doit conduire le conte moral.

Comme c'est le cas dans les anti-contes de fées écrits par Fénelon, la féerie est devenue un obstacle que le héros doit apprendre à surmonter.

Ces reprises viennent appuyer la répétition dans les deux contes de situations topiques. D'abord celle d'une femme tentée de fuir la réalité par les chimères de la fiction :

Ensuite la situation mondaine où hommes et femmes sont exposés à être la proie l'un de l'autre. Le mari de Séliane met garde Alcidonis contre la ruse des femmes tandis que le narrateur moraliste du Mari Sylphe revient à l'initiale du conte sur la justesse d'une pareille méfiance :

Évitez les pièges des hommes, dit-on sans cesse à une jeune femme : évitez la séduction des femmes, dit-on sans cesse à un jeune homme. Est-ce le plan de la nature que l'on croit suivre, en faisant d'un sexe l'ennemi de l'autre ? Ne sont-ils faits que pour se nuire 
? Sont-ils destinés à se fuir ? Et quel seroit le fruit de ces leçons, si tous les deux les prenoient à la lettre?

Les Quatres Flacons

Je suis bon pere : je ne veux que votre bonheur.

Le Mari Sylphe

La froideur impatiente \& dédaigneuse de sa femme détruisit cette illusion. Plus il étoit amoureux d'elle, plus il étoit blessé de l'éloignement qu'elle avoit pour lui ; \& cet amour si tendre \& si pur qui devoit faire son bonheur, alloit devenir son supplice.

Le conte revient sur la sagesse du conte de fées pour en contester la validité, pour en ruiner la moralité. Le conte de fées ne ferait que perpétuer des préjugés ancestraux qui attisent la guerre entre les sexes pendant que le conte moral s'applique au contraire à instaurer la paix. Le Mari Sylphe constitue bien un nouvel épisode de la métamorphose du conte de fées en conte moral.
Les Quatres Flacons
Dès qu'il m'ennuie, je le contredis ; il s'impatiente et se retire. l'on en fait tout ce qu'on veut. Je vous conseille de lui marquer de la déférence. Son amitié n'est pas à négliger : cela est bon à quelque chose.
Le Mari Sylphe
Il faut que la sensibilité de l'ame s'exerce ; \& si elle n'a pas un objet véritable, elle s'en fait un fantastique. Il étoit décidé dans l'opposition d'Élise, qu'il n'y avoit rien dans la nature qui fût digne de l'attacher. Mais elle avoit trouvé dans la fiction de quoi l'occuper, l'émouvoir, l'attendrir. La fable des Sylphes étoit à la mode. Il lui étoit tombé sous la main quelques-uns de ces romans où l'on peint le commerce délicieux de ces esprits avec les mortelles; \& pour elle ces brillantes chimeres avoient tout le charme de la vérité.

Dans ce conte, Marmontel choisit de nous éclairer sur les liens qu'il entend établir entre l'auteur et son lecteur dans les contes moraux, et cette relation, s'en étonnera-ton, est une relation morale qui présente, à ce titre, de graves incompatibilités avec l'immoralisme foncier de l'échange littéraire. L'avertissement est métafictionnel : Le Mari Sylphe est en effet la mise en fiction de l'échange littéraire entre un auteur illusionniste et un lecteur crédule qui, comme Élise, croit à l'existence d'une voix sans corps - le narrateur - prenant ici les traits de Valoé. Fiction du Sylphe Valoé, réalité du marquis de Volange, sous le regard enchanté d'Élise : le conte reproduit de façon ingénieuse son propre mode de fonctionnement et stipule la fonction qui incombe à chaque acteur de l'échange littéraire. Avec malice, Marmontel se plaît à entendre son personnage évoquer les pouvoirs qui sont les siens, à commencer par celui de lire dans la pensée de ses créatures :

Le Sylphe revint à son heure, \& parut charmé de lui voir saisir \& suivre ses idées avec tant de vivacité. Hélas! lui dit Elise, vous êtes plus heureux, vous devinez les miennes, \& vous sçavez les prévenir. Que le don de lire dans l'ame de ce qu'on aime est précieux! On ne lui donne pas le tems de désirer. Tel est sur moi votre avantage.

C'est encore le Sylphe-auteur qui inspire à Timothée des pensées et des vers dont il dit ignorer la source et dont il attribue la paternité à un " génie » :

Vous faites donc aussi des vers, lui demanda-t-elle en souriant? Ah! Madame, lui dit Timothée, c'est la chose du monde la plus singuliere, \& j'ai peine moi-même à la concevoir. J'avois oui dire qu'on avoit un génie, \& je prenois cela pour une fable ; mais ma foi rien n'est plus réel. J'en avois un, moi qui vous parle, \& je l'avois sans le savoir. Hier au soir encore je ne m'en doutois pas. - Et comment avez-vous fait cette découverte ? - Comment ? Cette nuit, dans le sommeil, en songe, mon génie 
m'est apparu \& m'a dicté les vers que voici : Je renonce au frivole honneur/De guider le char de l'Aurore, / D'annoncer le retour de Flore ; / Un soin plus doux fait mon bonheur : / Je préside au réveil de celle que j'adore. / L'Aurore a beau verser des pleurs, / L'Amante de Zephyre a beau semer des fleurs, / Elise est à mes yeux cent fois plus belle encore. Quoi! dit Elise toute émue, quoi! M. Timothée, vous avez fait ces vers! - Moi, Madame ! je n'en ai fait de ma vie. C'est mon génie qui me les a dictés. Il a fait plus : il les a mis en chant, \& vous allez voir comme il est habile... Hé-bien, Madame, dit-il après avoir chanté, que vous en semble ? N'est-on pas heureux d'avoir un génie comme le mien ? - Et, Monsieur, sçavez-vous du moins quelle est cette Elise que vous célébrez ? - Mais, Madame, je crois que c'est un nom comme Philis, Cloris, Iris. Mon génie a pris celui-là, parce qu'il est doux à l'oreille. - Ainsi, vous ne vous piquez pas d'entendre le sens des vers que vous chantez ? - Non, Madame, mais cela est égal : ils sont mélodieux, sensibles, \& ç'en est assez pour le chant. J'exige de vous, reprit-elle, qu'ils ne soient connus que de moi, \& si votre génie vous en inspire encore, je veux qu'ils me soient réservés.

51 Étrange déclaration que celle de ce personnage qui déclare ne rien comprendre à ce que l'auteur a placé dans sa bouche. Comme dans Jacques le fataliste, comme dans le théâtre de Pirandello, les créatures de papier s'animent et s'interrogent sur l'activité de leur créateur. Marmontel joue à divulger ces ficelles d'écrivain, notamment lorsqu'il compose la scène des bijoux entrelacés :

On se souvient qu'elle s'étoit amusée à tracer un chiffre où le nom de Valoé étoit enlacé dans le sien. Un jour qu'elle étoit invitée à une fête, elle voulut mettre ses diamans : elle ouvre son écrain, que voit-elle? ses bracelets, son collier, son aigrette, ses boucles d'oreille montés sur le dessein de ce chiffre qu'elle avoit tracé. Son premier sentiment fut celui de l'embarras \& de la surprise. Que va penser Volange ? Que va-t-il soupçonner ? Comme elle étoit encore à sa toilette, Volange arrive, \& jettant les yeux sur sa parure, Ah ! dit-il, rien n'est plus galant. Mon nom \& le vôtre dans un même chiffre ! Je serois bien flatté, Madame, que ce fût là un trait de sentiment! Elise rougit au lieu de feindre ; mais le soir Valoé fut grondé. Vous m'avez exposée, dit-elle, à un péril dont je tremble encore. J'ai vu le moment où il falloit que je trompasse mon mari, ou que je lui donnasse de moi l'idée la plus humiliante ; \& quoique l'avantage que tirent les hommes de notre sincérité nous autorise à la dissimulation, je sens qu'en usant de ce droit je serois mal avec moimême. Valoé ne manqua pas de louer cette délicatesse. Un petit mensonge, dit-il, est toujours un petit mal, \& je serois fâché d'en avoir été cause. Mais la ressemblance du nom de Volange avec le mien ne m'avoit point échappé, \& je savois que votre époux n'iroit pas plus loin que l'apparence. J'ai commencé par le rendre discret : c'est la premiere vertu d'un mari.

52 Marmontel s'explique ici sur la ressemblance trompeuse entre le narrateur et l'auteur, si nécessaire à l'illusion romanesque, ainsi que sur le judicieux retrait du narrateur qui fait passer auprès du lecteur la fiction comme une histoire qui semble se raconter d'elle-même.

53 Les Promenades de Platon présentent le même caractère métafictionnel et les objets magiques abandonnés à la contemplation du jeune Archèménès ne sont pas autre chose que des métaphores méta-énonciatives dans lesquelles l'auteur représente les modalités spécifiques de la création littéraire.

Procédons par ordre. Dans la bibliothèque des mages, où il découvre « le seul trésor échappé à [leur] ruine ", " des secrets merveilleux, des talismans, un art magique, qui est enseveli avec eux ", le prince admire d'abord le volume " où étaient déposées leurs études astrologiques, mais en lettres mystérieuses, inintelligibles pour [lui] ». Première prérogative de l'auteur : l'omniscience. Ensuite il contemple « un globe qui tournait sur 
son axe, sans aucun mobile au dehors ", "mécanisme d'un mouvement perpétuel comme celui des corps célestes, et au moyen duquel les arts pénibles n'auraient plus besoin d'ouvriers ». Deuxième prérogative de l'auteur: dissimuler le travail de composition et donner ainsi le caractère illusoire de réalité donnée à sa création fictionnelle. Ensuite encore l'attention est attirée par « le grand-œuvre, ou la pierre philosophale, le secret de changer en or tous les métaux ". Troisième prérogative de l'auteur : par le miracle de la métamorphose romanesque, donner à l'alliage impur de la vie humaine l'éclat précieux de la pureté amoureuse. Puis le mage lui présente une plante inconnue, " cette panacée que l'on cherche inutilement ", dont la " vertu serait de nous guérir de tous les maux et de prolonger nos années ». Quatrième prérogative de l'auteur : être maître du destin de ses créatures et pouvoir ménager en toute occasion une fin heureuse. Enfin le prince aperçoit un anneau d'or qu'il apprend être le " talisman du Lydien Gygès ». Quatrième et ultime prérogative de l'auteur : disparaître aux yeux des êtres de fiction comme à ceux de son lecteur et connaître les pensées de tous.

Omniscience universelle, dissimulo artis, sublimation romanesque d'une humanité imparfaite, omnipotence, ubiquité et connaissance des cœurs et des âmes : l'auteur est un dieu qui, dans l'exercice de ses pouvoirs que lui confèrent ses " rares inutilités ", cesse aussi d'être un homme. Le mage le signifie au jeune prince chaque fois que ce dernier s'indigne du secret gardé sur le trésor des mages :

Si cet art, me dit-il, avait été nécessaire au monde ; si seulement il avait eu plus d'utilité pour les hommes qu'il ne pouvait avoir d'abus et de dangers, nos pères l'auraient publié. Ils l'ont tenu caché ; et ce qui nous en reste, nous le cachons de même avec le plus grand soin. Car la nature a mesuré à l'homme l'intelligence, l'industrie et la force qu'il lui fallait pour son usage et pour son bien; et celui qu'elle daigne initier à ses mystères, la trahit en les divulguant.

Une fois encore, comme c'est la coutume chez Marmontel, la morale restreint la féerie et se donne, par extension, comme loi régulatrice de l'échange littéraire lui-même. Les Promenades de Platon tracent un sentier étroit entre deux précipices, d'une part celui féerique et romanesque de l'extravagance fictionnelle où l'auteur se joue de la crédulité $\mathrm{du}$ lecteur, et d'autre part celui critique et dysphorique de la divulgation des vérités intolérables qui composent le monde comme il va. Entre ces deux voies immorales, le conte moral nous engage dans un chemin médian, sans extravagance ni incrédulité, où le bonheur vertueux est à la mesure de la faiblesse humaine, où l'auteur ne se fonde pas sur le prestige de la fiction mais sur l'ascendant dont jouit la vertu sur tous les cœurs. $\mathrm{Au}$ terme de sa vie, Marmontel sera parvenu à moraliser la féerie, au moment même où s'annonce un regain du genre endormi, au moment où la morale rationnelle des Lumières est sur le point d'être supplantée par l'engouement du public pour les songes et les visions qui suscitent l'horreur et le frisson. Régression morale, aurait dit Marmontel mais, n'en déplaise au conteur, la littérature a ses raisons que la morale ignore. 


\section{NOTES}

1. Jean-François Marmontel, Mémoires, I, I, Paris, Mercure de France, 1999, p. 38 : « Je date d'un peu en parlant de mes bisaïeules ; mais je me souviens bien qu'à l'âge de quatre-vingts ans elles vivaient encore, buvant au coin du feu le petit coup de vin et se rappelant le vieux temps dont elles nous faisaient des contes merveilleux. »

2. Voir Régine Jomand-Baudry, "Préface », dans Le Conte merveilleux au XVIII siècle. Une poétique expérimentale, Paris, Kimé, 2002, p. 13-19). La tension est analysée en termes rhétoriques comme un divorce entre le placere et le docere.

3. Voir Anne Defrance, «Le conte de fées au risque de l'éloge politique : La Tyrannie des fées détruite (Mme d'Auneuil) et autres contes de la première génération ", dans Le Conte merveilleux au XVIII siècle. Une poétique expérimentale, ouvr. cité, p. 55-73.

4. Voir Raymonde Robert, Le Conte de fées en France de la fin du XVII siècle à la fin du XVIII siècle, Nancy, Presses universitaires de Nancy, 1981.

5. Voir Jean-Paul Sermain, «Les anti contes de fées de Fénelon », dans Conte merveilleux au XVIII siècle. Une poétique expérimentale, ouvr. cité, p. 243-250; Julie Bloch « Entre

convention et subversion : les contes de Caylus ", dans Le Conte merveilleux au XVIII siècle. Une poétique expérimentale, ouvr. cité, p. 42-54.

6. Voir Michel Delon, « Les Lumières et la dialectique du préjugé. L'exemple de $\mathrm{M}^{\mathrm{me}}$ de Staël », dans Frihetens Århundre. Litteratur, Kunst og filesoli i frankrike på 1700-tallet, Oslo, Spartacus Forlag, 1997, p. 68 ; repris dans Critique et légitimité du préjugé (XVIIl ${ }^{e}-X X^{e}$ siècle), Bruxelles, Éditions de l'Université de Bruxelles, 1999, p. 70.

7. Ibid., p. 65. Sur la conception nouvelle de la vérité comme jeu dialectique des opinions particulières, voir Nicolas Veysman, Mise en scène de l'opinion publique dans la littérature des Lumières, Paris, Champion, 2004.

8. Marmontel, Éléments de littérature, art. «Allégorie », ouvr. cité, p. 121.

9. « Le merveilleux surnaturel est tantôt l'image directe et simple, tantôt le voile symbolique et transparent de la vérité. Dans le premier cas, c'est la pure fiction ; dans le second, c'est l'allégorie ; mais ce n'est jamais que l'imitation exagérée de la nature.» 10. Jean-François Marmontel, Éléments de littérature, art. « Merveilleux », ouvr. cité, p. 716.

11. Kate Astbury, "Marmontel, éditeur du Mercure de France et de ses Contes moraux ", dans Marmontel : une rhétorique de l'apaisement, ouvr. cité, p. 162.

12. Jean Sgard, « Marmontel et la forme du conte moral », dans De l'Encyclopédie à la Contre-Révolution. Jean-François Marmontel (1723-1799), éd. Jean Ehrard, Clermont-Ferrand, G. de Bussac, 1970, p. 233.

13. Jean-François Marmontel, Poétique française, chapitre $X$.

14. Voir Pierre Pasquier, « Le merveilleux peut-il être merveilleux ? L'aporie de la crédibilité dans l'esthétique théâtrale classique ", dans Histoire de la France littéraire, Paris, PUF, 2006, t. II, p. 629-644.

15. Marc Escola, « Présentation » des Nouvelles galantes du XVII siècle, Paris, GF-Flammarion, 2004, p. 27.

16. Il le rappelle au chapitre XvIII de sa Poétique française (1763) : « La marche d'un poëme, quel qu'il soit, doit être celle de la nature, c'est-à-dire, telle qu'il nous soit facile de croire que les choses se sont passées comme nous les voyons. Or dans la nature les idées, les sentimens, les mouvemens de l'ame ont une génération qui ne peut être 
renversée sans un renversement de la nature même. Les événemens ont aussi une suite, une liaison que le poëte doit observer, s'il veut que l'illusion se soutienne. Des incidens détachés l'un de l'autre, ou mal-adroitement liés, n'ont plus aucune vraisemblance. Il en est du moral comme du physique, et du merveilleux comme du familier : pour que la contexture de la fable soit parfaite, il faut qu'elle ne tienne au-dehors que par un seul bout. Tous les incidens de l'intrigue doivent naître successivement l'un de l'autre, et c'est la continuité de la chaîne qui produit l'ordre et l'unité. »

17. Jan Herman, « "De quelle utilité peut-être le mensonge ?" ou le dilemme du roman ", dans Marmontel : une rhétorique de l'apaisement, Louvain-Paris, Éd. Peeters, 2003, p. 30. 18. Hélène Schrecker, «L'esprit sensible dans les œuvres de Marmontel », dans Roman et Lumières au XVIII siècle, Paris, Éd. sociales, 1970, p. 183.

19. Ibid., p. 186.

20. Jean-Paul Sermain, Le Conte de fées du classicisme aux Lumières, Paris, Desjonquères, 2005, p. 184.

21. Voir Jan Herman « Pour une définition ex negativo du conte. Le statut du conte dans les préfaces de romans de la première moitié du XvIII ${ }^{e}$ siècle », in Le Conte merveilleux au XVIII siècle. Une poétique expérimentale, ouvr. cité, p. 74-86.

\section{RÉSUMÉS}

De 1755 à 1792, Marmontel écrit des contes moraux que nous disons être les héritiers des contes de fées. Cette étude s'attache en effet à mettre en évidence l'existence d'une féerie morale, d'abord par l'existence d'objets magiques et de personnages féeriques, mais aussi par la présence de structures narratives communes. Le féerique ne devient toutefois véritablement moral qu'avec la mise en scène de prodiges moraux où ce n'est plus le surnaturel mais la vertu naturelle qui provoque la stupéfaction et permet de surmonter tous les obstacles. Les objets et les personnages jouent cependant un rôle déterminant chez Marmontel, puisqu'ils sont autant d'indices métafictionnels grâce auxquels l'auteur mène une réflexion sur la moralité de la féerie, et, plus généralement, sur la moralité de la création littéraire.

\section{Moral fairy in Marmontel's tales :}

From 1755 to 1792, Marmontel wrote moral tales that we consider as inherited from fairy tales. This study aims at enhancing the existence of a moral fairy, first through the existence of magic objetcs and fairy-like characters, and also as well as through the presence of common storytelling structures. The fairy only becomes truly moral when astonishment is produced through with the staging of moral prodigies thanks to which asthonishment is provoqued and when the obstacles are overcome no longer by the supernatural but by the natural virtue. The Oobjects and characters, however, play a prominent part in Marmontel's works since they represent as many metafictional clues, thanks to which the author carries out a reflection about the morality of fairy and, more generally, about the morality of literary creation. 